\title{
Development of Indium Phosphide MEMS for Tunable Optical Buffering
}

\author{
Nina Podoliak ${ }^{1}$, Wing H. Ng${ }^{2}$, Will Stewart ${ }^{1}$, Huiyun Liu ${ }^{2}$, Anthony J. Kenyon ${ }^{2}$, Peter Horak ${ }^{1 *}$ \\ ${ }^{1}$ Optoelectronics Research Centre, University of Southampton, Southampton SO17 1BJ, United Kingdom \\ ${ }^{2}$ Department of Electronic and Electrical Engineering, University College London, London WC1E 7JE, United \\ Kingdom. \\ *e-mail:peh@orc.soton.ac.uk
}

\begin{abstract}
We are developing a MEMS-based device that can produce continuously tunable delays of optical signals. The design is based on coupled dual suspended waveguides fabricated in Indium Phosphide. We report on simulation-based optimisation of optical and mechanical properties of the structure and on current progress in fabrication.
\end{abstract}

Keywords: Microelectromechanical systems, optical buffering, optical waveguides, III-V semiconductor materials, indium phosphide.

\section{INTRODUCTION}

Optical buffering is a key functionality in all-optical data processing, allowing for delay or storage of optical pulses without the need for conversion into the electronic format. Practical technical solutions are however still limited: fixed-fibre delay lines are bulky [1], while small-footprint resonator-based solutions typically only operate over very narrow bandwidths $[2,3]$.

Our approach to developing an integrated optical buffer is based on mechanical reconfiguration of coupled dual semiconductor optical waveguides [4]. The fundamental operating principle of this device is illustrated in Fig. 1. We consider two sub-wavelength sized dielectric waveguides (of InP material, refractive index 3.17 at $1550 \mathrm{~nm}$ wavelength, size $195 \times 300 \mathrm{~nm}$ ) separated by an air gap of size $g$. At large $g=500 \mathrm{~nm}$, Fig. 1(a), the two waveguides support a fundamental mode that spreads widely into air and thus has an effective mode index close to 1 . Bringing the waveguides closer together by mechanical actuation, e.g. to $g=50 \mathrm{~nm}$ in Fig. 1(b), leads to much stronger confinement of the optical mode inside the high-index semiconductor material and thus the effective mode index increases significantly and, in other words, the speed of the propagating light decreases. Our simulations have shown that this argument not only holds for the phase velocity of light but also for the group velocity, such that the device can achieve continuously tunable delay by a factor of 2 to 3 for optical pulses operating over a very wide bandwidth in excess of $100 \mathrm{~nm}$ [4].

(a)

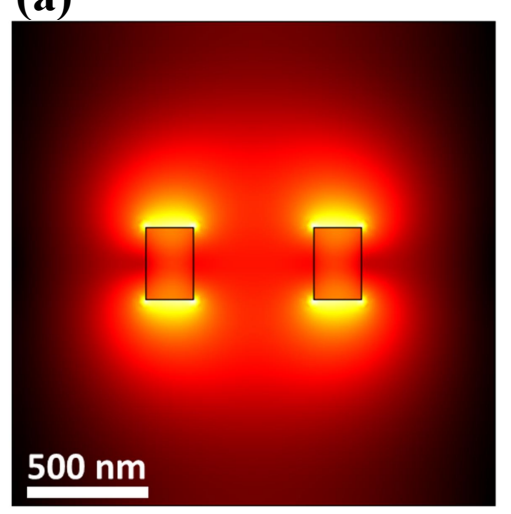

(b)

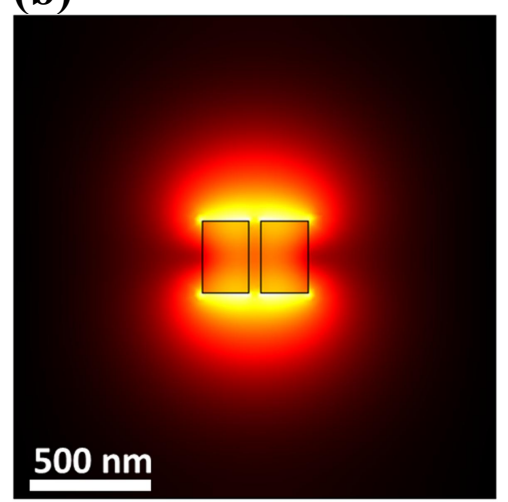

Figure 1. Operation principle of the continuously tunable optical buffer. The fundamental optical mode (colour indicates modulus of electric field) supported by the two high-index waveguides changes significantly when the air gap between the waveguides is reduced from (a) $\mathrm{g}=500 \mathrm{~nm}$ to (b) $50 \mathrm{~nm}$, leading to a continuously tunable speed of light.

Careful design optimisation and fabrication is required to turn this concept into a working integrated device: the individual waveguides need to be mechanically supported at regular distances chosen to allow for easy mechanical actuation while providing sufficient structural support; microelectromechanical systems (MEMS) type actuation via incorporated electrodes on the chip must provide for large mechanical displacements while avoiding pull-in and sticking effects; optical propagation losses must be kept at a minimum or compensated by optical gain. Because of this latter requirement we have chosen to work in direct bandgap InP material. This will 
allow for integration of optical amplification on the same chip as the optical buffer. On the other hand, InP is far less established for MEMS than for example silicon and we have put significant efforts into developing this technology [5].

\section{DESIGN OPTIMISATION}

\subsection{Waveguide dimensions}

The width and height of the optical waveguides are chosen to maximise the change in group index of the fundamental optical mode when the waveguides are moved relative to each other (Fig. 1). For InP this is achieved with rectangular waveguides of width $w=195 \mathrm{~nm}$ and height $h=300 \mathrm{~nm}$ [5].

\subsection{Supporting pillars}

The air-suspended dual waveguides need to be suspended at regular intervals $L$. According to the EulerBernoulli beam equations the maximum displacement $d$ of a waveguide under a given uniform force $F$ (in $\mathrm{N} / \mathrm{m}$ ) and the mechanical oscillation frequency $f$ are given by

$$
d=\frac{F L^{4}}{32 E h w^{3}} \text { and } f=\frac{5.6}{\pi} \frac{w}{L^{2}} \sqrt{\frac{E}{3 \rho}},
$$

respectively, where $E=89 \mathrm{GPa}$ is Young's modulus and $\rho=4800 \mathrm{~kg} / \mathrm{m}^{3}$ is the density of InP [6]. Thus large pillar separations $L$ allow for actuation with lower forces while, on the other hand, they also lead to a low mechanical oscillation frequency and thus to slow response times and sensitivity to vibration noise. A pillar separation of $100 \mu \mathrm{m}$ is chosen as a compromise (mechanical oscillation frequency $f=86 \mathrm{kHz}$ ).

\subsection{Propagation losses}

Various mechanisms contribute to the optical losses experienced by propagating light in the dual suspended waveguides. These need to be minimised and managed carefully for a practical device.

- Surface roughness of the waveguides causes light scattering and a corresponding loss rate of $2.2 \mathrm{~dB} / \mathrm{cm}$ was reported for InP waveguides [7], while lower losses have been measured in silicon.

- Supporting pillars cause scattering where they connect to the waveguides. For our waveguides we found by finite element simulations that a simple T-shaped connection induces losses of $\sim 1 \mathrm{~dB}$ per pillar, which can be reduced to $0.2 \mathrm{~dB}$ when a tapered connection is used [5].

- Evanescent fields in the air around the InP waveguides carry a significant fraction of the optical power in our device, in particular at large waveguide separations. While this is essential for the continuous effective index tunability it can also lead to coupling of light out of the waveguides into any nearby materials, e.g. the chip substrate. Our simulations indicate that these losses are below $0.1 \mathrm{~dB} / \mathrm{cm}$ for waveguide-surface separations larger than $3 \mu \mathrm{m}$.

- Free carrier absorption is observed when the waveguides are doped to enable electric conductivity, as required for the electrostatic actuation discussed below. We estimate that doping concentrations of $5 \times 10^{17} \mathrm{~cm}^{-3}$ induce losses of $2.5 \mathrm{~dB} / \mathrm{cm}$, i.e. comparable to the intrinsic surface roughness losses.

\section{ACTUATION}

We considered two different electrostatic actuation methods for our device. In the first method the waveguides are undoped, thus reducing the free carrier absorption losses discussed above. Additional electrodes implemented on the chip are used to generate non-uniform electrostatic fields that polarise the waveguides. The interaction of these induced dipoles with the background field and the dipole-dipole interaction between the two waveguides then generate mechanical forces [8]. However, relatively large voltages of up to $50 \mathrm{~V}$ are required in this case [5].

The second method of mechanical actuation assumes that voltages are directly applied to the two waveguides, which thus have to be doped to be electrically conducting. Finite element simulations of Maxwell's stress tensor where the same voltage $V$ and polarity is applied to two rectangular waveguides are used to calculate the mechanical forces on the waveguides, see Fig. 2(a). Note that the forces scale with $V^{2}$. Together with Eq. (1), these results can be used to calculate the stationary waveguide displacement under electrostatic actuation where the electrostatic forces balance the elastic mechanical forces, shown in Fig. 2(b). For a pillar spacing of $100 \mu \mathrm{m}$, about $4 \mathrm{~V}$ are required to increase the air gap between the two waveguides from $50 \mathrm{~nm}$ to $500 \mathrm{~nm}$. The voltages required to achieve the same waveguide displacement for different pillar spacing scales approximately as $1 / L^{2}$. Note that by applying the same voltage to the two waveguides, actuation relies on repulsive forces only, which helps to avoid pull-in effects and waveguide sticking. 
(a)

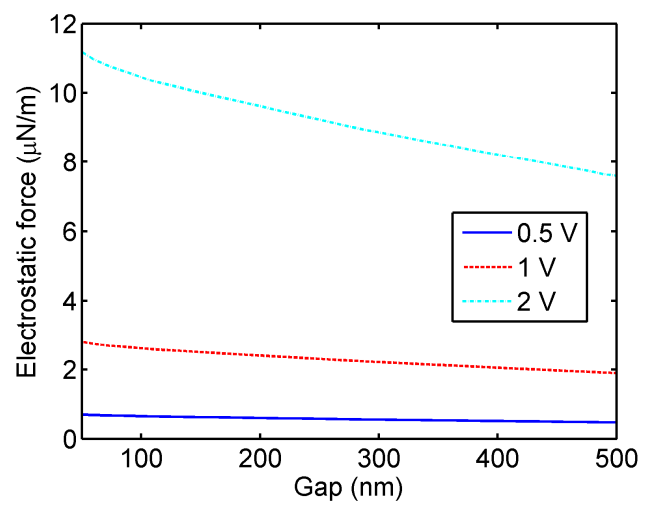

(b)

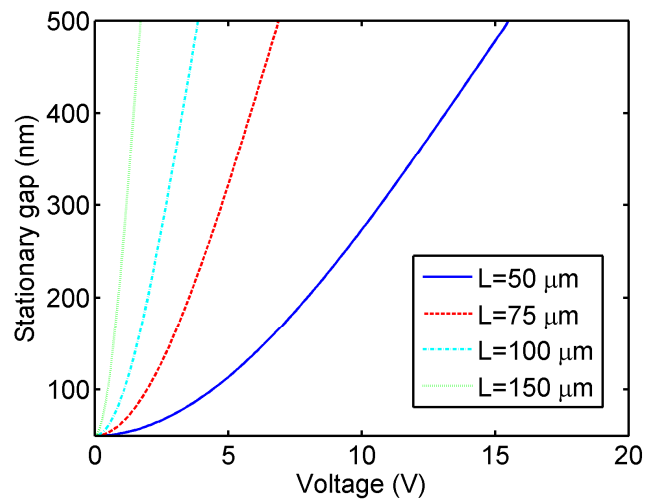

Figure 2. (a) Electrostatic force on each waveguide for fixed applied voltages versus air gap size. (b) Stationary gap size where electrostatic forces are balanced by elastic forces versus applied voltage for different supporting pillar spacing $L$.

\section{FABRICATION}

For fabrication of the device designed above, we first grow the sample layer structure shown in Fig. 3(a) by molecular beam epitaxy. The InGaAs layer acts as a sacrificial layer that is etched away in a later step to release the waveguides. The waveguides themselves are made of InP with a $1 \%$ admixture of Ga to provide a small amount of tensile stress for mechanical stability. The waveguides and pillar structures are patterned by hydrogen silsesquioxane electron beam resist masks and etched by reactive ion etching. Finally, the waveguides are released by etching with $\mathrm{HF}: \mathrm{H}_{2} \mathrm{O}_{2}: \mathrm{H}_{2} \mathrm{O}(1: 1: 8)$ solution and subsequent supercritical drying with $\mathrm{CO}_{2}$.

(a)

\begin{tabular}{|l|}
\hline Waveguide Layer: $\operatorname{In}_{0.99} \mathrm{Ga}_{0.01} \mathrm{P}$ \\
\hline Sacrificial Layer: $\operatorname{In}_{0.53} \mathrm{Ga}_{0.47} \mathrm{As}$ \\
\hline Substrate: n-type $\operatorname{InP}$ \\
\hline
\end{tabular}

(b)

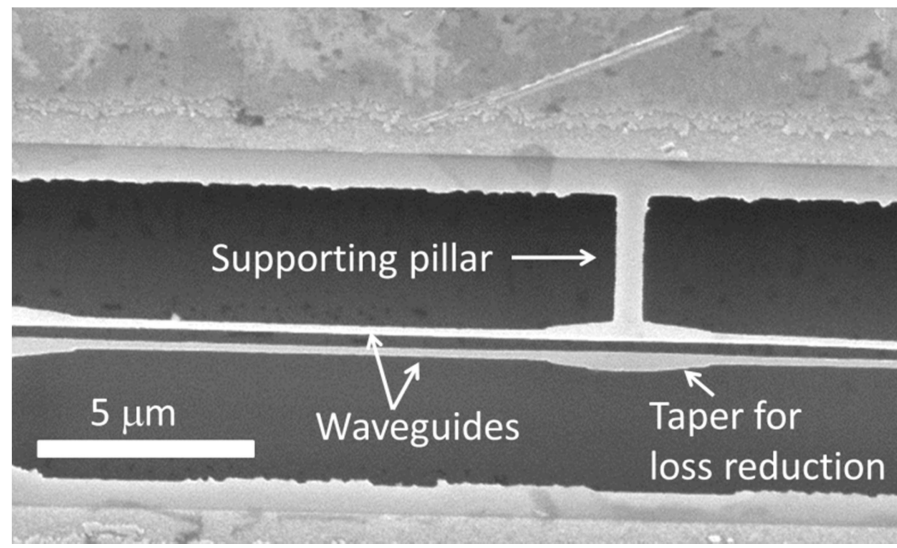

Figure 3. (a) Schematic of the sample layer structure. (b) SEM image of the fabricated optical buffer structure.

A scanning electron microscope (SEM) image of the fabricated structure is shown in Fig. 3(b). One can clearly see the dual suspended waveguide structure with an air gap between them, the tapered section of the waveguides required to reduce scattering losses at the pillar support points as discussed in Sec. 2.3, and the supporting pillars themselves. The structure at the top of the image is plated with a gold layer to act as a metal electrode pad required to apply voltages to the waveguides.

\section{CONCLUSIONS}

We have designed and fabricated an optical MEMS device in III-V semiconductor material (InP) to act as an optical buffer that allows for continuously tunable speed of light by a factor of two. Following on theoretical and numerical device simulation and optimisation, we have developed a protocol for fabrication of the buffer device. Our current fabricated samples are not yet fully realising the optimised design (air gap too large, short pillar spacing), but all the required ingredients for a practical and working device have been demonstrated. Once the fabrication has been perfected, we will aim to demonstrate the mechanical actuation and tunable buffer functionality. 


\section{ACKNOWLEDGEMENTS}

This work was supported by the Engineering and Physical Sciences Research Council under Grant EP/J012823/1 and EP/J012874/1. The data used in this publication is available at [9] under the Creative Commons Attribution 4.0 License.

\section{REFERENCES}

[1] R. Langenhorst, et al.: Fiber loop optical buffer, J. Lightwave Technol., vol. 14, pp. 324-335, 1996.

[2] F. Xia, L. Sekaric, Y. Vlasov: Ultracompact optical buffers on a silicon chip, Nat. Photon., vol. 1, pp. 6571, 2007.

[3] N. K. Fontaine, et al:: Continuously tunable optical buffering at $40 \mathrm{~Gb} / \mathrm{s}$ for optical packet switching networks, J. Lightwave Technol., vol. 26, pp. 3776-3783, 2008.

[4] P. Horak, W. Stewart, W. H. Loh: Continuously tunable optical buffer with a dual silicon waveguide design, Opt. Express, vol. 19, pp. 12456-12461, 2011.

[5] W. H. Ng, N. Podoliak, P. Horak, J. Wu, H. Liu, W. J. Stewart, A. J. Kenyon: Design and fabrication of suspended indium phosphide waveguides for MEMS-actuated optical buffering, IEEE J. Sel. Topics Quantum Electron., vol. 21, article no. 4400107, 2015.

[6] M. J. Weber: Handbook of Optical Materials, Boca Raton: CRC Press, 2003.

[7] D. Kelly, et al.: Monolithic suspended optical waveguides for InP MEMS, IEEE Photon. Technol. Lett., vol. 16, pp. 1298-1300, 2004.

[8] N. Podoliak, Z. Lian, W.H. Loh, P.Horak: Design of dual-core optical fibers with NEMS functionality, Opt. Express, vol. 22, pp. 1065-1076, 2014.

[9] N. Podoliak, W. H. Ng, W. Stewart, H. Liu, A. J. Kenyon, P. Horak: Development of Indium Phosphide MEMS for tunable optical buffering, dataset, University of Southampton eprints, http://eprints.soton.ac.uk/id/eprint/376157; doi: 10.5258/SOTON/376157. 